TITLE:

\title{
Theory of exciton thermal radiation in semiconducting single-walled carbon nanotubes
}

$\operatorname{AUTHOR}(\mathrm{S})$ :

Konabe, Satoru; Nishihara, Taishi; Miyauchi, Yuhei

\section{CITATION:}

Konabe, Satoru ... [et al]. Theory of exciton thermal radiation in semiconducting singlewalled carbon nanotubes. Optics Letters 2021, 46(13): 3021-3024

ISSUE DATE:

2021-07

URL:

http://hdl.handle.net/2433/267482

RIGHT:

(C) 2021 Optical Society of America under the terms of the OSA Open Access Publishing Agreement 


\title{
Theory of exciton thermal radiation in semiconducting single-walled carbon nanotubes
}

\author{
Satoru Konabe, ${ }^{1, *}$ (1) Taishi Nishihara, ${ }^{2}$ (1) and Yuhel Miyauchi ${ }^{2}$ \\ ${ }^{1}$ Department of Chemical Science and Technology, Hosei University, Tokyo 184-8584, Japan \\ ${ }^{2}$ Institute of Advanced Energy, Kyoto University, Kyoto 611-0011, Japan \\ *Corresponding author: konabe@hosei.ac.jp
}

Received 28 April 2021; revised 27 May 2021; accepted 31 May 2021; posted 1 June 2021 (Doc. ID 430011 ); published 17 June 2021

\begin{abstract}
Spectral control of thermal radiation is an essential strategy for highly efficient and functional utilization of thermal radiation energy. Among the various proposed methods, quantum confinement in low-dimensional materials is promising because of its inherent ability to emit narrowband thermal radiation. Here, we theoretically investigate thermal radiation from one-dimensional (1D) semiconductors characterized by the strong quantum correlation effect due to the Coulomb interaction. We derive a simple and useful formula for the emissivity, which is then used to calculate the thermal radiation spectrum of semiconducting single-walled carbon nanotubes as a representative of 1D semiconductors. The calculations show that the exciton state, which is an electron-hole pair mutually bound by the Coulomb interaction, causes enhancement of the radiation spectrum peak and significant narrowing of its linewidth in the near-infrared wavelength range. The theory developed here will be a firm foundation for exciton thermal radiation in 1D semiconductors, which is expected to lead to new energy harvesting technologies. () 2021 Optical Society of America under the terms of the OSA Open Access Publishing Agreement
\end{abstract}

https://doi.org/10.1364/OL.430011

Thermal radiation is a ubiquitous form of electromagnetic radiation that emanates from objects with finite temperature, and its spectrum can be described by Planck's law [1] modified by the absorptivity of the object (Kirchhoff s law [2]). Absorptivity control of objects using micro- and nanofabrication techniques has recently attracted increasing attention [3,4] due to the importance of thermal radiation control in energy applications such as thermophotovoltaic (TPV) energy conversion [5-29], thermal management of spacecraft [30], and daytime radiative cooling [31] of buildings [32] and human clothing $[33,34]$. TPV technology [5-10], which utilizes tailored narrowband near-infrared thermal radiation from hot objects that well matches the bandgap energy of a photovoltaic (PV) cell as an input, is of particular importance in the realization of high-efficiency energy harvesting from high-temperature heat sources. When sunlight is used as a heat source for an ideal TPV system, the theoretical energy conversion efficiency of the solar TPV can even surpass the Shockley-Queisser limit [35] for a single-junction PV cell [6]. To generate spectrally narrowband near-infrared thermal radiation in the far-field [5-17] and near-field regions [21-25], a number of surface microstructures of refractory metals have been demonstrated using metamaterials [10-13], microcavities [14,15], and photonic crystals $[16,17]$. Although, in principle, metal surfaces with elaborate fine structures possess a photonic bandgap and exhibit a sharp resonance [36], their thermal stability is inevitably reduced. Consequently, high-temperature operation requires the use of more thermally stable, simpler structures that can offer only imperfect spectral control. As an alternative approach, atomic emission lines in the infrared-to-visible region, such as those for rare-earth-doped ceramics, are attractive in terms of generating spectrally narrowband thermal radiation [27,28]. However, their resonance energies are not tunable, and their linewidths are too narrow, which results in low in-band power density [26,29].

Spectral control that relies on electronic structures in semiconductors has also attracted much attention. For a semiconductor photonic crystal, both the photonic bandgap and the electronic bandgap make strong contributions to fine spectral control [18-20]. The use of exciton resonance in semiconducting single-walled carbon nanotubes (SWCNTs) has also been recently proposed as a new approach [37]. A SWCNT is a cylindrically rolled-up graphene sheet, and it is regarded as a quasi-one-dimensional (1D) system with a pronounced quantum confinement effect. Narrowband near-infrared thermal radiation from an individual semiconducting SWCNT was recently reported [37]. This was enabled by the thermal generation of excitons, which are exotic atoms composed of a mutually bound electron-hole pairs. Because an exciton captures the considerable oscillator strength of graphene [38-40], the dielectric function has a very large imaginary part of a few to several tens at the exciton resonance, with a moderately narrow spectral window [41]. In addition, the exciton resonance is tunable by control of the structure (the diameter and/or chiral angle), which is specified by two integers known as the chiral indices $(n, m)$. Furthermore, SWCNT excitons and SWCNTs themselves are stable even at temperatures as high as $2000 \mathrm{~K}$ [37], due to their large binding energies [38-40] and stable $s p^{2}$ covalent bonding [42], respectively. These features are quite promising for the use of SWCNTs under high-temperature conditions. Although the narrowband thermal radiation from SWCNTs may prove to be particularly useful for TPV technologies, a detailed understanding in the presence of the strong quantum 
correlation effect characteristic of 1D materials, including the exciton effects, has yet to be achieved. In the present Letter, we theoretically elucidate the thermal radiation properties of SWCNTs as a representative of $1 \mathrm{D}$ materials by developing a microscopic quantum theory of thermal radiation with the Coulomb interaction effect.

We begin by providing a useful formula for the emissivity of $1 \mathrm{D}$ cylindrical objects. The thermal radiation from a material at temperature $T, H(T)$, is generally characterized by the spectral emissivity $e(\omega)$ :

$$
H(T)=\frac{\hbar}{4 \pi^{2} c^{2}} \int_{0}^{\infty} \mathrm{d} \omega e(\omega) \frac{\omega^{3}}{e^{\hbar \omega / k_{\mathrm{B}} T}-1},
$$

where $\omega$ is the angular frequency of the radiation, $c$ is the speed of light, $\hbar=h / 2 \pi$ is the reduced Planck constant, and $k_{\mathrm{B}}$ is the Boltzmann constant. The emissivity depends on size and shape for materials smaller than or comparable in size to the thermal wavelength $\lambda=\hbar c / k_{\mathrm{B}} T$. For such cases, the thermal radiation is given by the following trace formula derived by Krüger $e t a l$. [43] in the framework of fluctuational electrodynamics [44]:

$$
\begin{aligned}
H(T)= & \frac{2 \hbar}{\pi} \int_{0}^{\infty} \mathrm{d} \omega \frac{\omega}{e^{\hbar \omega / k_{\mathrm{B}} T}-1} \\
& \times \operatorname{Tr}\left[\operatorname{Im}\left(\mathbf{G}_{\mathbf{0}}\right) \operatorname{Im}(\mathbf{T})-\operatorname{Im}\left(\mathbf{G}_{\mathbf{0}}\right) \mathbf{T} \operatorname{Im}\left(\mathbf{G}_{\mathbf{0}}\right) \mathbf{T}^{*}\right] .
\end{aligned}
$$

Here we define

$$
e(\omega) \equiv \frac{8 \pi c^{2}}{\omega^{2}} \operatorname{Tr}\left[\operatorname{Im}\left(\mathbf{G}_{\mathbf{0}}\right) \operatorname{Im}(\mathbf{T})-\operatorname{Im}\left(\mathbf{G}_{\mathbf{0}}\right) \mathbf{T} \operatorname{Im}\left(\mathbf{G}_{\mathbf{0}}\right) \mathbf{T}^{*}\right],
$$

so that the conventional formula for thermal radiation is reproduced as Eq. (1). In the following, we thus use the term "emissivity" to describe $e(\omega)$. In Eq. (3), $\mathbf{G}_{0}$ is the dyadic Green's function for the electromagnetic field in free space. The scattering operator $\mathbf{T}=\mathbf{V} \frac{1}{1-\mathbf{G}_{0} \mathbf{V}}$ [45] relates the electromagnetic field in free space to that of the inhomogeneous space with the object characterized by the potential $\mathbf{V}$, where $\mathbf{V}=\frac{\omega^{2}}{c^{2}}(\hat{\varepsilon}-\mathbf{I}) ; \hat{\varepsilon}$ and $\mathbf{I}$ are the dielectric tensor and identity matrix, respectively. $\mathbf{T}$ causes dissipation of electromagnetic energy. $\mathbf{G}_{0}$ and $\mathbf{T}$ can be calculated for a $1 \mathrm{D}$ object by imposing the boundary condition of a cylindrical structure on the electromagnetic wave [43,46-48]. Under the long wavelength limit of $\omega d / c \ll 1$, Eq. (3) in the vicinity of the resonance becomes

$$
e(\omega) \simeq \frac{\omega d}{3 c} \operatorname{Im}[\varepsilon(\omega)],
$$

where $d$ is the diameter of the cylindrical object, and $\varepsilon(\omega)$ is the dielectric function along the cylinder axis (see also Supplement $1)$. The thermal radiation from any $1 \mathrm{D}$ material that satisfies the condition $\omega d / c \ll 1$ using (4) can be calculated as long as the dielectric function of the material is obtained.

The thermal radiation from SWCNTs as a representative of $1 \mathrm{D}$ materials is investigated using Eqs. (1) and (4) with a focus on the peculiar electronic excitation of SWCNTs. The electronic structure around the bandgap energy of SWCNTs (see the inset in Fig. 1 for the selection of the coordinate system) is effectively described by the following Hamiltonian [38]:

$$
\mathcal{H}_{\lambda}=\gamma\left(\kappa_{l \nu}^{\lambda} \sigma_{x}+\lambda k \sigma_{y}\right),
$$

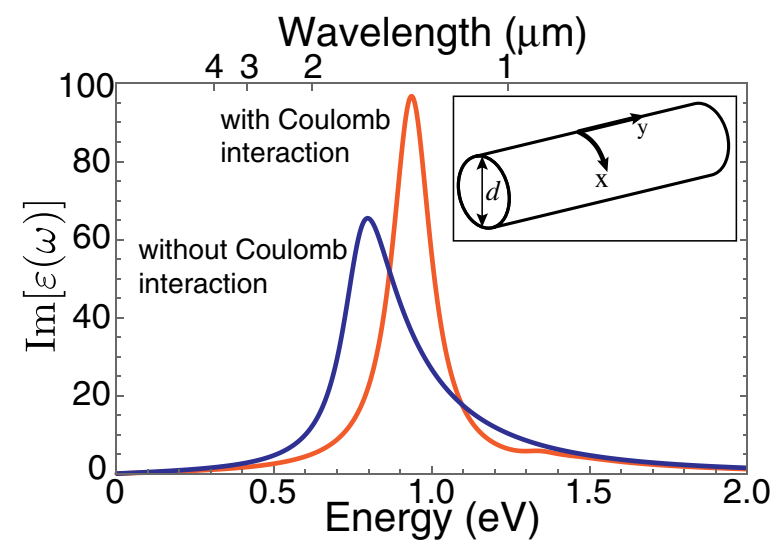

Fig. 1. $\operatorname{Im}[\varepsilon(\omega)]$ for a $1.12 \mathrm{~nm}$ diameter SWCNT. Broadening is set to $75 \mathrm{meV}$. Inset: $1 \mathrm{D}$ cylindrical model and coordinate system of a SWCNT.

where $\sigma_{x}$ and $\sigma_{y}$ are the Pauli matrices, $\lambda= \pm 1$ is an index that labels the $K$ or $K^{\prime}$ points in the Brillouin zone for graphene, and $\gamma=0.646 \mathrm{eV} \mathrm{nm}$ is a band parameter. The first (second) term on the right-hand side describes electron motion with a wave vector $\kappa_{l v}^{\lambda}(k)$ in the circumference (tube axis) direction $[x(y)$ axis]. As a result of the periodic boundary condition along the circumference direction, the wave vector for a $(n, m)$ SWCNT is quantized as $\kappa_{l v}^{\lambda}=2(l-\lambda v / 3) / d$, where $v \equiv \bmod (n-m, 3)$ is the chirality, and $l$ is an integer that represents the energy band. The energy band is obtained by diagonalizing Eq. (5) as $\varepsilon_{c / v, k}^{\lambda}= \pm \sqrt{\gamma^{2}\left[k^{2}+\left(\kappa_{l v}^{\lambda}\right)^{2}\right]}$, where $+(-)$ indicates the conduction (valence) band, which is denoted as $\varepsilon_{c k}^{\lambda}\left(\varepsilon_{v k}^{\lambda}\right)$.

Furthermore, the Coulomb interaction effect for 1D materials such as SWCNTs should be incorporated in the electronic structure because the Coulomb interaction is less well screened than that in bulk materials. As a result of the attractive Coulomb interaction between an electron and a hole, a bound state of the electron and hole, an exciton, is formed. The $r$ th exciton state with amplitude $\psi_{k, r}^{\lambda}$ and energy $E_{r}^{\lambda}$ satisfies the Bethe-Salpeter equation $[38,49-51]$

$$
\left(\varepsilon_{c k}^{\lambda}-\varepsilon_{v k}^{\lambda}+\Delta_{k}^{\lambda}\right) \psi_{k, r}^{\lambda}+\sum_{k^{\prime}} \mathcal{K}_{k, k^{\prime}}^{\lambda} \psi_{k^{\prime}, r}^{\lambda}=E_{r}^{\lambda} \psi_{k, r}^{\lambda},
$$

where $\Delta_{k}^{\lambda}$ is the band-energy shift due to the electron-electron repulsive Coulomb interaction, and $\mathcal{K}_{k, k^{\prime}}^{\lambda}$ is the electron-hole Coulomb interaction. We incorporate the screening effect of the Coulomb interaction in solving the Bethe-Salpeter equation to reproduce the experimental values of the exciton binding energy. For a SWCNT with a diameter of $1.12 \mathrm{~nm}$, the exciton energy is $1.0 \mathrm{eV}$ with a binding energy of $0.5 \mathrm{eV}$. A large binding energy of an exciton is characteristic of SWCNTs [38-40,50].

The dielectric function is calculated from the dynamical conductivity through the relation $\varepsilon(\omega)=1+i \sigma(\omega) / \varepsilon_{0} \omega$. The dynamical conductivity of SWCNTs is obtained using the Kubo formula [38]

$$
\sigma(\omega)=\frac{32 e^{2} \hbar^{2} \omega}{\pi A d^{2}} \sum_{\lambda} \sum_{r} \frac{\left|M_{r}^{\lambda}\right|^{2}}{E_{r}^{\lambda}\left[\left(E_{r}^{\lambda}\right)^{2}-(\hbar \omega)^{2}-2 i \hbar \omega \Gamma\right]},
$$


where $A$ is the tube length, $M_{r}^{\lambda}$ is the optical matrix element between the ground state and the $r$ th exciton state, and $\Gamma$ is the phenomenological broadening factor. The optical matrix element is explicitly given by $M_{r}^{\lambda}=i \frac{\gamma}{\hbar} \sum_{l, k} \frac{\kappa_{l v}^{\lambda}}{\sqrt{k^{2}+\left(\kappa_{l v}^{\lambda}\right)^{2}}}\left(\psi_{k, r}^{\lambda}\right)^{*}$. The noninteracting limit of the dynamical conductivity is calculated by using $\psi_{k, r}^{\lambda}=\delta_{r, k}$ and $E_{r}^{\lambda}=2 \sqrt{\gamma^{2}\left[k^{2}+\left(\kappa_{l v}^{\lambda}\right)^{2}\right]}$ in Eq. (7).

Figure 1 plots $\operatorname{Im}[\varepsilon(\omega)]$ for a SWCNT with a diameter of $1.12 \mathrm{~nm}$. The linewidth of the dynamical conductivity is set to $75 \mathrm{meV}$, which is consistent with the experimental value at high temperature $(1300 \mathrm{~K})$ given in Ref. [37]. In the presence of the Coulomb interaction, $\operatorname{Im}[\varepsilon(\omega)]$ exhibits a sharp and almost symmetric peak that corresponds to the lowest exciton state (red curve), which is in contrast to a broad asymmetric peak structure with a high energy tail due to the $1 \mathrm{D}$ van Hove singularity, in the absence of the Coulomb interaction (blue curve). In addition, the continuum above the bandgap $(>1.4 \mathrm{eV})$ has a small weight because considerable optical intensity is transferred to the exciton state as expected in a $1 \mathrm{D}$ material $[38,52]$. The peak height of the exciton is consequently larger than that without the Coulomb interaction.

Figures 2(a) and 2(b) show the emissivities for SWCNTs with various diameters, calculated using Eq. (4) for cases with and without the Coulomb interaction, respectively. The resonance energy of the emissivity is dependent on the diameter. The peak position of the emissivity is accordingly tunable by controlling the diameter. The important findings are that the linewidth is narrower and the height is a few times larger for an exciton than those for a free electron-hole pair. Therefore, the presence of an exciton due to the Coulomb interaction qualitatively and quantitatively alters the emissivity. We also plot the emissivity for graphene [53] to observe the dimensional effect. The broadly dispersed spectral weights for graphene become concentrated at a specific photon energy for SWCNTs, which results in an enhancement of the spectral intensity for SWCNTs.

Note that the contribution of exciton-ionized electrons to the emissivity can be neglected because excitons are stable due to their large binding energies of several hundred millielectronvolts and do not dissociate, even at temperatures much higher than room temperature. On the other hand, for a free electron-hole pair, there is typically a contribution from the intraband electron excitation, i.e., the Drude contribution. However, the present calculation does not take account of the Drude contribution; therefore, the calculation result for a free electron-hole pair in Fig. 2(b) should be regarded as providing an upper limit.

Having clarified the characteristic features of the emissivity, we will investigate the thermal radiation. Figure 3 shows the spectral radiance with a resonance wavelength of $1.3 \mu \mathrm{m}$ at 1300 $\mathrm{K}$. For practical use, the horizontal axis is set to wavelength. For comparison, blackbody radiation at the same temperature is shown by a dashed line, which is scaled by 0.18 . From the results for the emissivity, thermal radiation in the case of a Coulomb interaction can be identified as due to excitons, while thermal radiation without a Coulomb interaction is due to free electronhole pairs. For both cases, the thermal radiation from SWCNTs has a much narrower linewidth than blackbody radiation. In particular, the linewidth of exciton thermal radiation is narrower than that of thermal radiation from free electron-hole pairs. Furthermore, the exciton thermal radiation is a few times larger than the thermal radiation from free electron-hole pairs. We thus conclude that the Coulomb interaction plays a decisive role in the thermal radiation from SWCNTs [37], which may be true for other 1D materials as well. The inset of Fig. 3 shows that the peak position of thermal radiation shifts to a longer wavelength as the diameter increases. This indicates that the resonance energy of the thermal radiation of SWCNTs is tunable by the simple selection of an appropriate diameter.

Before concluding, we note that excitons are typically observed under photo- and electrical-excitation conditions. Therefore, it is reasonable to consider whether the exciton state can be thermally generated at $1300 \mathrm{~K}$. A simple model can be constructed where the exciton is generated via excitonmultiphonon interaction, which is given in Supplement 1 .

In summary, thermal radiation from 1D semiconductors was theoretically studied, where the Coulomb interaction plays a crucial role, by deriving a simple and useful formula for the emissivity. The formula was used to calculate the thermal radiation spectrum of SWCNTs as a representative of $1 \mathrm{D}$ semiconductors. Our calculation shows that, due to the exciton state, the peak of the radiation spectrum is enhanced and the spectral shape becomes more symmetric and narrower in the near-infrared wavelength range. The peak position of the thermal radiation is determined by the diameter of SWCNTs; therefore, the desired wavelength of thermal radiation can be selected simply by choosing an appropriate diameter. The results obtained in this Letter will be useful for the development of wavelength selective thermal emitters based on SWCNTs toward high-efficiency TPV energy conversion.

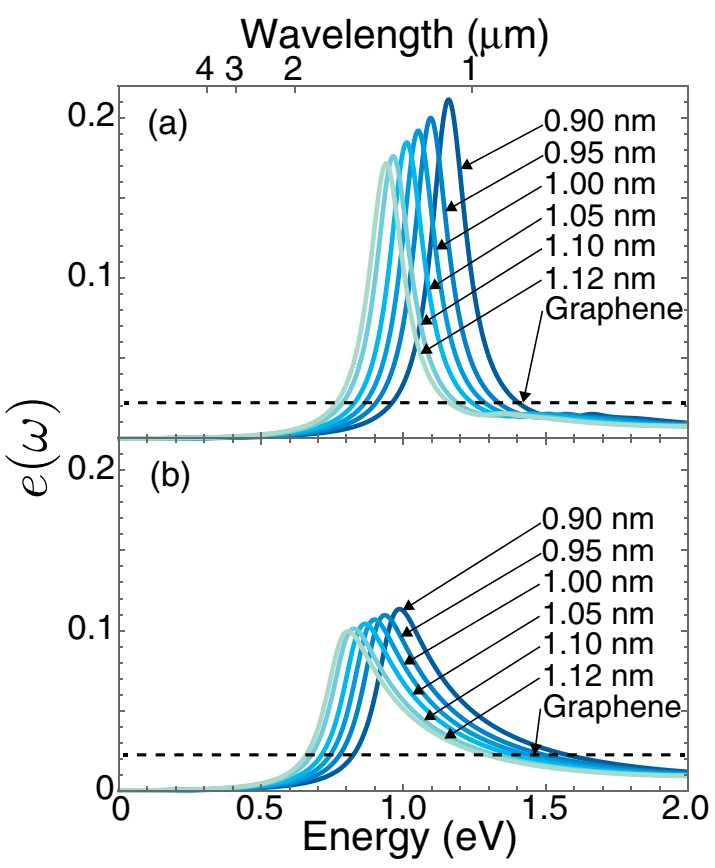

Fig. 2. Emissivity (a) with and (b) without the Coulomb interaction as a function of energy for SWCNTs with diameters of 0.90, 0.95, $1.00,1.05,1.10$, and $1.12 \mathrm{~nm}$. 


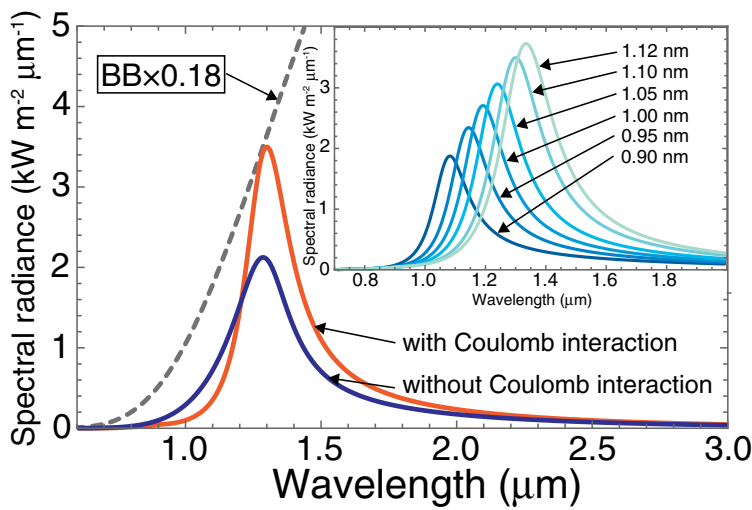

Fig. 3. Spectral radiance for a SWCNT at $1300 \mathrm{~K}$, with and without Coulomb interaction. The black dashed line represents blackbody radiation at $1300 \mathrm{~K}$. Note that the blackbody radiation is scaled by 0.18 , and the spectra for SWCNTs with different diameters showing the same peak wavelength are compared. Inset: spectral radiance for SWCNTs with various diameters.

Funding. Japan Society for the Promotion of Science (JP16H06331, JP19K15384, JP20H02605, JP20H05664); Core Research for Evolutional Science and Technology (JPMJCR18I5).

Acknowledgment. This work is supported by the ZE Research Program, IAE(ZE2020B-33, ZE2021B-02).

Disclosures. The authors declare no conflicts of interest.

Data Availability. Data underlying the results presented in this Letter are not publicly available at this time but may be obtained from the authors upon reasonable request.

Supplemental document. See Supplement 1 for supporting content.

\section{REFERENCES}

1. M. Planck, Ann. Phys.309, 553 (1901).

2. G. Kirchhoff, Philos. Mag. Ser.20(20), 1 (1860).

3. W. Li and S. Fan, Opt. Express26, 15995 (2018).

4. D. G. Baranov, Y. Xiao, I. A. Nechepurenko, A. Alù, and M. A. Kats, Nat. Mater.18, 920 (2019).

5. E. Rephaeli and S. Fan, Opt. Express17, 15145 (2009).

6. A. Datas and A. Mart, Solar Energy Mater. Sol. Cells 161, 285 (2017).

7. A. Lenert, D. M. Bierman, Y. Nam, W. R. Chan, I. Celanovic, M. Soljačić, and E. N. Wang, Nat. Nanotechnol.9, 126 (2014).

8. D. M. Bierman, A. Lenert, W. R. Chan, B. Bhatia, I. Celanovic, M. Soljačić, and E. N. Wang, Nat. Energy1, 16068 (2016).

9. A. Kohiyama, M. Shimizu, and H. Yugami, Appl. Phys. Express9, $112302(2016)$

10. D. N. Woolf, E. A. Kadlec, D. Bethke, A. D. Grine, J. J. Nogan, J. G. Cederberg, D. B. Burckel, T. S. Luk, E. A. Shaner, and J. M. Hensley, Optica 5, 213 (2018).

11. S. Molesky, C. J. Dewalt, and Z. Jacob, Opt. Express21, A96 (2013).

12. D. Woolf, J. Hensley, J. G. Cederberg, D. T. Bethke, A. D. Grine, and E. A. Shaner, Appl. Phys. Lett.105, 081110 (2014).

13. P. N. Dyachenko, S. Molesky, A. Y. Petrov, M. Störmer, T. Krekeler, S. Lang, M. Ritter, Z. Jacob, and M. Eich, Nat. Commun.7, 11809 (2016).

14. S. Maruyama, T. Kashiwa, H. Yugami, and M. Esashi, Appl. Phys. Lett.79, 1393 (2001).

15. F. Kusunoki, T. Kohama, T. Hiroshima, S. Fukumoto, J. Takahara, and T. Kobayashi, Jpn. J. Appl. Phys.43, 5253 (2004).

16. Y. X. Yeng, M. Ghebrebrhan, P. Bermel, W. R. Chan, J. D. Joannopoulos, M. Soljačić, and I. Celanovic, Proc. Natl. Acad. Sci. USA109, 2280 (2012).

17. K. A. Arpin, M. D. Losego, A. N. Cloud, H. Ning, J. Mallek, N. P. Sergeant, L. Zhu, Z. Yu, B. Kalanyan, G. N. Parsons, G. S. Girolami, J. R. Abelson, S. Fan, and P. V. Braun, Nat. Commun.4, 2630 (2013).
18. T. Asano, M. Suemitsu, K. Hashimoto, M. De Zoysa, T. Shibahara, T. Tsutsumi, and S. Noda, Sci. Adv. 2, e1600499 (2016).

19. M. Suemitsu, T. Asano, M. De Zoysa, and S. Noda, Appl. Phys. Lett.112, 011103 (2018).

20. M. Suemitsu, T. Asano, T. Inoue, and S. Noda, ACS Photon.7, 80 (2020).

21. J. C. Cuevas and F. J. Garcia-Vidal, ACS Photon.5, 3896 (2018).

22. Y. Guo, C. L. Cortes, S. Molesky, and Z. Jacob, Appl. Phys. Lett.101, 131106 (2012).

23. K. Isobe, D. Hirashima, and K. Hanamura, Int. J. Heat Mass Transfer 115, 467 (2017).

24. A. Fiorino, L. Zhu, D. Thompson, R. Mittapally, P. Reddy, and E. Meyhofer, Nat. Nanotechnol.13, 806 (2018)

25. T. Inoue, T. Koyama, D. D. Kang, K. Ikeda, T. Asano, and S. Noda, Nano Lett.19, 3948 (2019).

26. R. Sakakibara, V. Stelmakh, W. R. Chan, M. Ghebrebrhan, J. D. Joannopoulos, M. Soljačić, and I. Celanovic, J. Photon. Energy $\mathbf{9}, 1$ (2019).

27. N. Nakagawa, H. Ohtsubo, Y. Waku, and H. Yugami, J. Eur. Ceram. Soc. 25,1285 (2005).

28. A. Manor, N. Kruger, T. Sabapathy, and C. Rotschild, Nat. Commun.7, 510 (2016).

29. G. Torsello, M. Lomascolo, A. Licciulli, D. Diso, S. Tundo, and M. Mazzer, Nat. Mater.3, 632 (2004).

30. H. Demiryont and D. Moorehead, Solar Energy Mater. Sol. Cells 93 , 2075 (2009).

31. B. Zhao, M. Hu, X. Ao, N. Chen, and G. Pei, Appl. Energy236, 489 (2019).

32. X. Lu, P. Xu, H. Wang, T. Yang, and J. Hou, Renew. Sustain. Energy Rev.65, 1079 (2016).

33. P. C. Hsu, A. Y. Song, P. B. Catrysse, C. Liu, Y. Peng, J. Xie, S. Fan, and Y. Cui, Science 353, 1019 (2016).

34. X. A. Zhang, S. Yu, B. Xu, M. Li, Z. Peng, Y. Wang, S. Deng, X. Wu, Z. Wu, M. Ouyang, and Y. Wang, Science 363, 619 (2019).

35. W. Shockley and H. J. Queisser, J. Appl. Phys.32, 510 (1961).

36. X. Liu, T. Tyler, T. Starr, A. F. Starr, N. M. Jokerst, and W. J. Padilla, Phys. Rev. Lett.107, 045901 (2011).

37. T. Nishihara, A. Takakura, Y. Miyauchi, and K. Itami, Nat. Commun.9, 3144 (2019).

38. T. Ando, J. Phys. Soc. Jpn.66, 1066 (1997).

39. F. Wang, G. Dukovic, L. E. Brus, and T. F. Heinz, Science 308, 838 (2005).

40. J. Maultzsch, R. Pomraenke, S. Reich, E. Chang, D. Prezzi, A. Ruini, E. Molinari, M. S. Strano, C. Thomsen, and C. Lienau, Phys. Rev. B72, 6680 (2005).

41. F. Yao, C. Liu, C. Chen, S. Zhang, Q. Zhao, F. Xiao, M. Wu, J. Li, P. Gao, J. Zhao, X. Bai, S. Maruyama, D. Yu, E. Wang, Z. Sun, J. Zhang, F. Wang, and K. Liu, Nat. Commun.9, 3387 (2018).

42. K. M. Liew, C. H. Wong, X. Q. He, and M. J. Tan, Phys. Rev. B71, $075424(2005)$

43. M. Krüger, G. Bimonte, T. Emig, and M. Kardar, Phys. Rev. B86, 115423 (2012).

44. S. M. Rytov, Y. A. Kravtsov, and V. I. Tatarskii, Principles of Statistical Radiophysics (Springer-Verlag, 1989), Vol. 3.

45. S. J. Rahi, T. Emig, N. Graham, R. L. Jaffe, and M. Kardar, Phys. Rev. D80, 085021 (2009).

46. E. Noruzifar, T. Emig, and R. Zandi, Phys. Rev. A84, 39 (2011).

47. M. Krüger, T. Emig, and M. Kardar, Phys. Rev. Lett.106, 210404 (2011).

48. V. A. Golyk, M. Krüger, and M. Kardar, Phys. Rev. E85, 046603 (2012).

49. V. Perebeinos, J. Tersoff, and P. Avouris, Phys. Rev. Lett.92, 257402 (2004).

50. C. D. Spataru, S. Ismail-Beigi, L. X. Benedict, and S. G. Louie, Phys. Rev. Lett.92, 077402 (2004).

51. J. Jiang, R. Saito, G. G. Samsonidze, A. Jorio, S. G. Chou, G. Dresselhaus, and M. S. Dresselhaus, Phys. Rev. B75, 035407 (2007).

52. T. Ogawa and T. Takagahara, Phys. Rev. B44, 8138 (1991).

53. R. Nair, P. Blake, A. N. Grigorenko, K. S. Novoselov, T. J. Booth, T. Stauber, N. M. R. Peres, and A. K. Geim, Science 320, 1308 (2008). 